\title{
Regulatory and Ethical Approval Timelines for HIV Vaccine Studies: An Analysis of International AIDS Vaccine Initiative (IAVI) Sponsored Studies in East and Southern Africa
}

\section{Prince Bahati ${ }^{1 *}$, Zablon Omungo ${ }^{1}$, Bonnie Bender ${ }^{1}$ and Josea Rono ${ }^{2}$}

${ }^{1}$ International AIDS Vaccine Initiative, Nairobi, Kenya

${ }^{2}$ E\&K Consulting Firm LLP, Nairobi, Kenya

\begin{abstract}
Background: There has not been a systematic analysis of factors affecting ethics and regulatory timelines of HIV Vaccine trials and epidemiologic studies in Eastern and Southern Africa. We analyzed regulatory and ethics approval timelines and associated factors for HIV vaccine clinical trials and epidemiologic studies in Kenya, Uganda, Rwanda, South Africa and Zambia using data collected from seven Clinical Research Centers (CRCs) from 2001 to 2015.

Methodology: Staff responsible for regulatory issues at CRCs provided archived data on ethical and regulatory review time-frames which were then validated with the sponsor central database. A semi structured questionnaires was administered to establish qualitative information on perceived factors affecting efficiency of approval processes and potential solutions. Quantitative data analysis was conducted using Excel. Qualitative data were analyzed using an open coding to analyze and elicit general themes.

Findings: Data on submission for 23 clinical trials and 51 epidemiological study protocols were analyzed. Across all seven CRCs it took on average of 178 and 108 days to obtain full authorization to commence clinical trials and epidemiological studies, respectively. These timelines are shorter than the average found in other disease fields in Africa but seem longer than approval timelines in the USA and the EU that are estimated at 15-45 days and 43-75 days, respectively. We found that countries with sequential, rather than parallel, submission procedures had longer review timelines. Clinical trial approval timelines that were longer than 200 days were associated with amendment submissions prior to initial approval and with investigational products that required institutional biosafety committee reviews. Lengthier approval timelines were also associated with epidemiological studies with more invasive procedures.
\end{abstract}

Conclusion: Strategies to further shorten timelines will need to focus on adoption of parallel approval processes; increase frequency of ethical review meetings and capacity strengthening of ethical review institutions.

Keywords: Ethical review timelines in Africa; Efficiency of review and regulatory processes in Africa; HIV vaccine ethics and regulatory issues

\section{Introduction}

Emerging markets in and out of Africa are hosting an increasing number of health research studies that use human subjects. About $40 \%$ of clinical trials are currently being conducted in Low and Middle Income Countries (LMIC) in Asia, Latin America and Africa [1]. This has been mainly attributed to the great burden of disease in these countries and also, thus in part, making it comparatively less expensive to conduct these studies in LMICs [2] and the increasing difficulty in finding sufficient numbers of eligible study participants in more developed economies [3].

While conducting health research in Africa is important to the global research community and is beneficial to Africa [1], the efficiency with which these health research studies are conducted in Africa is increasingly becoming an important question because of both the public health cost related to delayed access to health products and increased costs related to longer timelines of health research. These timelines affect the overall efficiency of conducting health research, the overall cost of health research [4] and the productivity of pharmaceutical industries, including vaccine manufacturers [5]. Paradoxically, despite the influence of approval timelines on the efficiency of health research, there has been little systematic evaluation of the timelines of existing ethical and regulatory processes.

Timelines are dependent on numerous factors, such as the complexity of the proposed study, the capacity of regulatory and review bodies, approval policies and procedures, the quality of the submitted proposal and the efficiency of the researcher in responding to internal review boards (IRB) queries. To generate insights into the approval timelines of HIV vaccine clinical trials and epidemiological studies in Africa, the International AIDS Vaccine Initiative (IAVI) conducted a qualitative and quantitative study of regulatory and ethical approval timelines at seven partner clinical research centers (CRCs) that have been conducting HIV vaccine clinical trials and epidemiological studies in Africa since 2001. The research centers are: Project San Francisco (PSF) in Rwanda; Zambia Emory HIV Research Project (ZEHRP) in Zambia; Aurum Institute in South Africa; Kenya AIDS Vaccine Initiative - Institute for Clinical Research (KAVI-ICR) and Kenya Medical Research Institute (KEMRI) - Wellcome Trust Research Programme in Kenya; and Uganda Virus Research Institute-

${ }^{*}$ Corresponding author: Prince Bahati, International AIDS Vaccine Initiative Nairobi, Kenya, Tel: +254719043000; +254707367951; E-mail: PBahati@iavi.org

Received February 24, 2017; Accepted March 09, 2017; Published March 16, 2017

Citation: Bahati P, Omungo Z, Bender B, Rono J (2017) Regulatory and Ethical Approval Timelines for HIV Vaccine Studies: An Analysis of International AIDS Vaccine Initiative (IAVI) Sponsored Studies in East and Southern Africa. J AIDS Clin Res 8: 676. doi: 10.4172/2155-6113.1000676

Copyright: @ 2017 Bahati P, et al. This is an open-access article distributed under the terms of the Creative Commons Attribution License, which permits unrestricted use, distribution, and reproduction in any medium, provided the original author and source are credited. 
International AIDS Vaccine Initiative HIV Vaccine Program (UVRIIAVI) and Medical Research Council/Uganda Virus Research Institute (MRC/UVRI) in Uganda. We present the findings of IAVI's assessment and discuss them in light of existing literature on the approval timelines for health research in Africa, factors affecting approval timelines and strategies that have contributed to improvements in these timelines.

\section{Methods}

The study was based on archival data generated in the course of implementing HIV clinical trials and epidemiological studies at the seven IAVI-sponsored CRCs between 2001 and 2015. Staff responsible for regulatory issues at IAVI partner CRCs provided data on ethical and regulatory review time-frames for past and current clinical trials and observational Studies. They also completed semi structured questionnaires to establish qualitative information on perceived factors affecting efficiency of approval processes and potential solutions. Quantitative data analysis was conducted using Excel. Qualitative data from the questionnaire were analyzed using an open coding to analyze and elicit general themes.

\section{Data collection}

Staff in charge of regulatory issues across the seven IAVI partner CRCs provided data on ethical and regulatory review time-frames for past and current clinical trials and observational studies. The questionnaires also included open-ended and semi-structured questions that were used to collect qualitative data on factors affecting approval timelines and potential strategies that can contribute to the shortening of these timelines. Due to specialization of ethics and regulatory functions at individual CRCs, the CRC staff in-charge of the institutional review board (IRB) docket at the CRC was targeted as the main respondent and the $\mathrm{CRC}$ directors as co-signatory to the data.

\section{Data analysis}

We analyzed secondary data from archives databases at IAVI and CRCs on documented approval timelines for epidemiologic studies and clinical trials. An open-ended and semi-structured questionnaire was sent to the CRC staff in charge of IRB docket to identify factors that affected timelines, trends and potential solutions in increasing efficiency of review timelines. Quantitative data analysis was conducted using Microsoft Excel 2010 software. Approval timelines were computed as the difference in days between the date when a clinical trial or epidemiological study protocol was submitted for review and the date when full authorization to commence the trial or study was granted. Qualitative data on factors that affected length of review were also collected from the questionnaire and was analyzed using an open coding to analyze and create general themes [6].

\section{Results}

\section{Approval timelines across clinical research centers}

A total of 23 clinical trial protocol submissions were analyzed. This included one submission from Aurum institute, two from ZEHRP, three from MRC/UVRI, five from PSF and 6 each from KAVI-ICR and UVRI-IAVI. Overall, across all the six CRCs (there were no clinical trial data from KEMRI Wellcome Trust Research Programme); it took an average of 178 days to obtain full authorization to commence clinical trials. On average it took 70 days to receive full authorization to commence clinical trials at PSF (126 days if approvals from an affiliated US university institutional review board are considered), 140 days at MRC/UVRI, 186 days at Aurum institute, 204 days at UVRI-IAVI, 212 days at KAVI-ICR and 284 days at ZEHRP (Figure 1).

Fifty-one epidemiological study protocol submissions were analyzed. This number was made up of two submissions at Aurum Institute, six at KEMRI-Wellcome Trust Research Programme, six at UVRI-IAVI, seven at ZEHRP, eight at PSF, 10 at MRC/UVRI and 12 at KAVI. Across all the seven CRCs, full approval to commence epidemiological studies was obtained after 108 days. On average approval of protocol submissions took 59, 87, 90, 107, 111, 123 and 213 days at PSF, MRC-UVRI, ZEHRP, KAVI-ICR, UVRI-IAVI, Aurum Institute and -KEMRI Wellcome Trust Research Programme, respectively (Figure 2).

\section{Comparison of approval timelines of specific clinical trials and epidemiological studies across CRCs}

A detailed comparison of specific HIV vaccine clinical trials across individual CRCs and countries is presented in. While not all clinical trials were conducted in all six CRCs, it is worth noting that approval trials for clinical trials conducted at PSF are shorter compared to approval timelines for the same trials conducted in other CRCs. For instance, full approval for clinical trial HIV-V-004/J\&J_I was obtained in 48 days at PSF while approval for the same clinical trial at UVRIIAVI took 121 days. In another instance, full approval for clinical trial

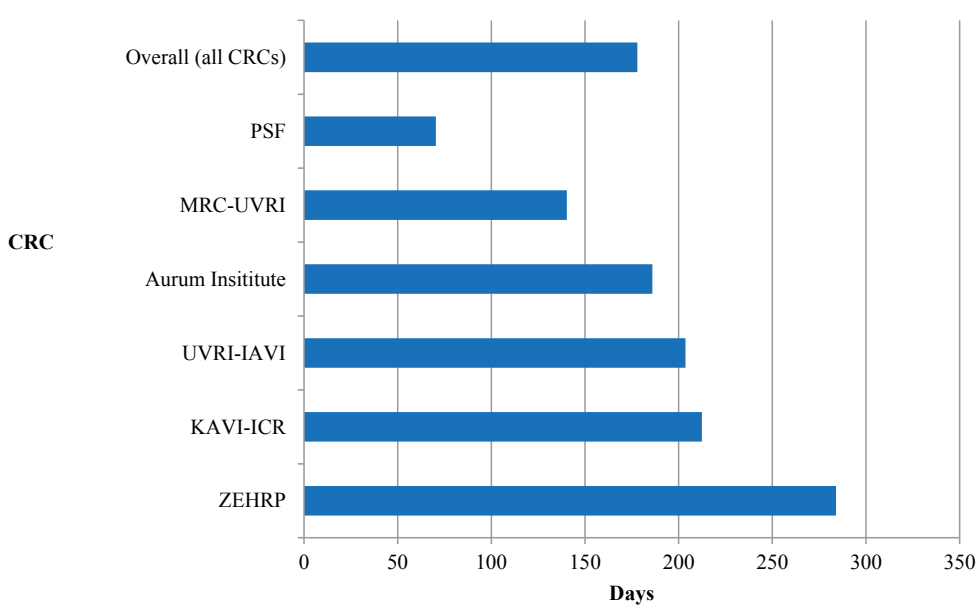

Figure 1: Average approval timelines for regulatory and ethics review for clinical trial protocols at PSF, ZEHRP, Aurum Institute, MRC-UVRI, UVRI-IAVI and KAVI-ICR. 


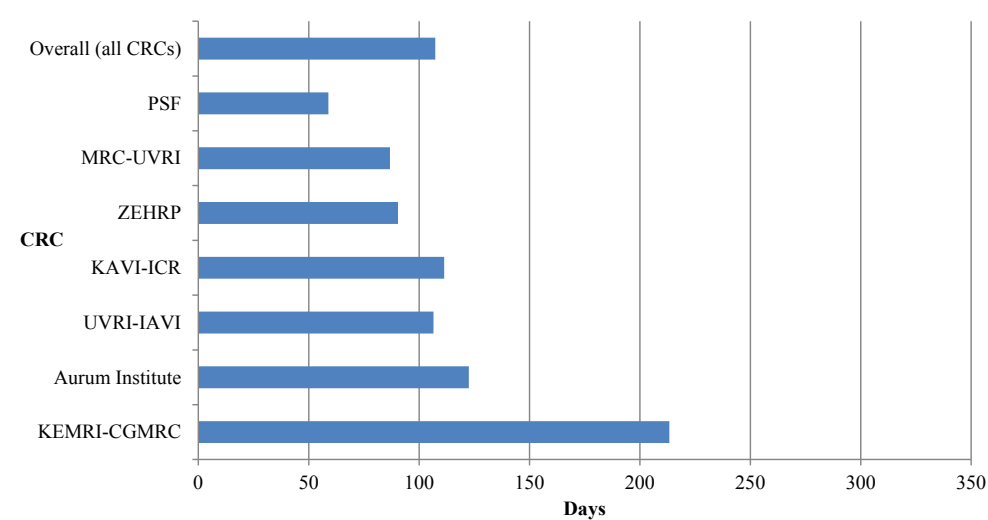

Figure 2: Average approval timelines for regulatory and ethics review for epidemiological study protocols at PSF, ZEHRP, Aurum Institute, MRC-UVRI, UVRI-IAVI, KEMRI-CGMRC and KAVI-ICR.

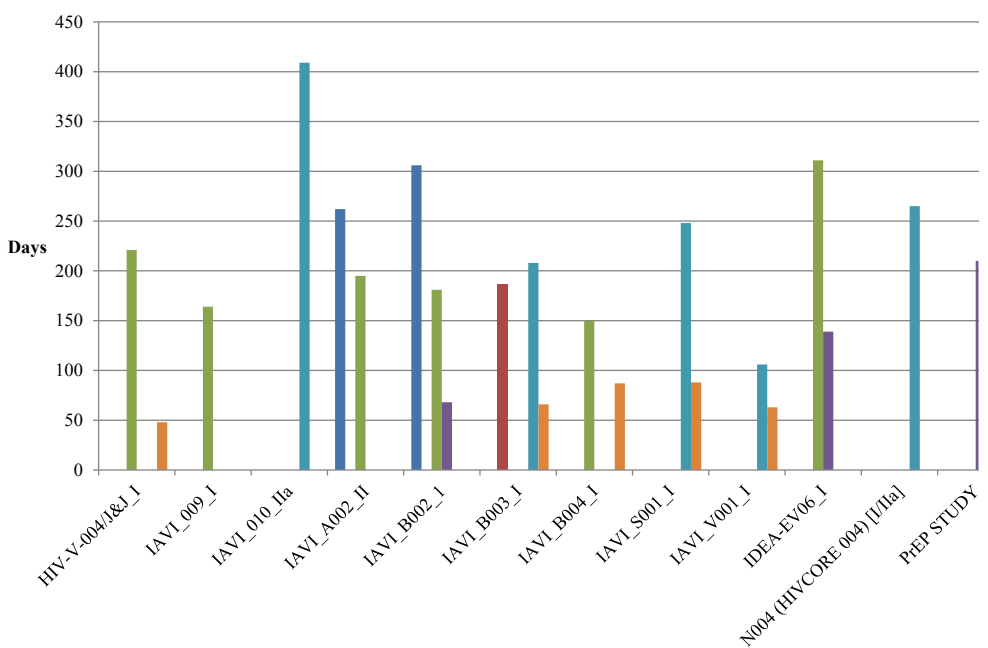

Clinical trial

Figure 3: Comparison of approval timelines for individual clinical trials protocols across different CRCs.

IAVI_B003_I was granted after 66 days at PSF yet approval for the same trial at Aurum Institute and KAVI-ICR took 186 and 208 days respectively. Similar evidence of shorter approval timelines at PSF compared to other CRCs is demonstrated by data relating to clinical trials IAVI_S001_I and IAVI_V001_I (Figure 3).

Similarly, to the data on clinical trials, a detailed comparison of specific epidemiological studies across the seven CRCs suggests that approval timelines are generally shorter at PSF compared to other CRCs. For instance, while approval for comparison of approval timelines for Protocol L epidemiological study at PSF was granted 38 days after protocol submission, approval for the same study conducted at KAVIICR and MRC/UVRI took 188 and 393 days respectively. Similar evidence of shorter approval timelines at PSF compared to other CRCs is demonstrated by data relating to protocol D and $\mathrm{G}$ epidemiological studies (Figure 4).

\section{Temporal trends in approval timelines}

Assessment of temporal trends in approval timelines of regulatory and ethical review of clinical trial protocols in the six CRCs showed that, taking all CRCs together, there is no evidence of a change in approval timelines between 2001 and 2014. Nonetheless, CRC-specific trends suggest that approval timelines have lengthened at some CRCs such as ZEHRP and KAVI-ICR; shortened at other CRCs such as MRC-UVRI; and remained relatively unchanged at other CRCs such as PSF (Figure 5).

Temporal trend analysis of approval timelines of ethical and regulatory review for HIV epidemiological studies seem to suggest that, taking data from all CRCs together, approval timelines have lengthened between 2001 and 2015. Similarly, to the data on clinical trials, CRCspecific trends suggest that approval timelines for epidemiological studies have lengthened at some CRCs such as UVRI-IAVI, MRC/ UVRI, KAVI-ICR and KEMRI-Wellcome Trust Research Programme while they have shortened at other CRCs such as PSF (Figure 6).

\section{Qualitative feedback on temporal trends in approval timelines}

Qualitative responses submitted by CRCs as to whether approval timelines for regulatory and ethical approval processes for clinical trials and epidemiological studies have reduced over time were mixed and varied by country. Despite data showing increase in review timelines, the majority of respondents felt like overall review timelines have improved. Earlier studies may have been less complex than later studies and they cautioned comparing review timelines across all clinical trials, epidemiologic and target cohorts as the same. 


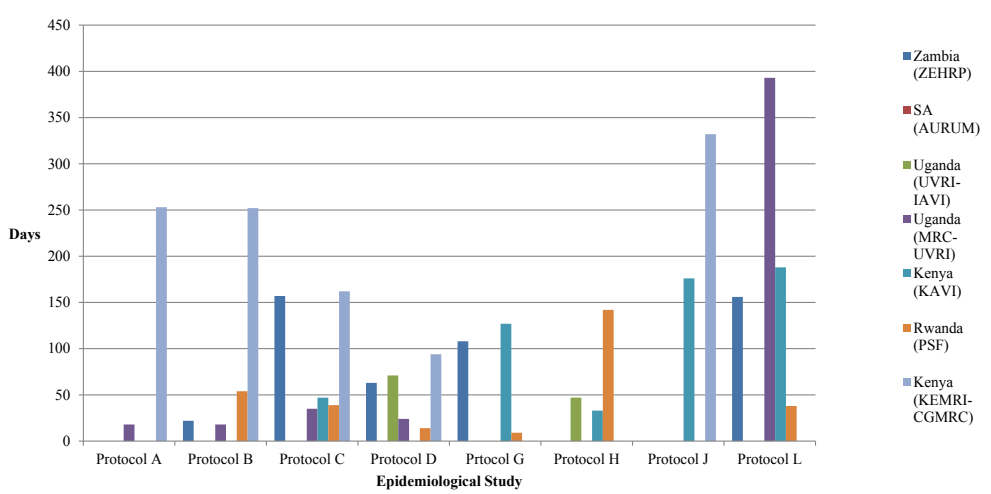

Figure 4: Comparison of approval timelines for individual and specific epidemiological study protocols across different CRCs.

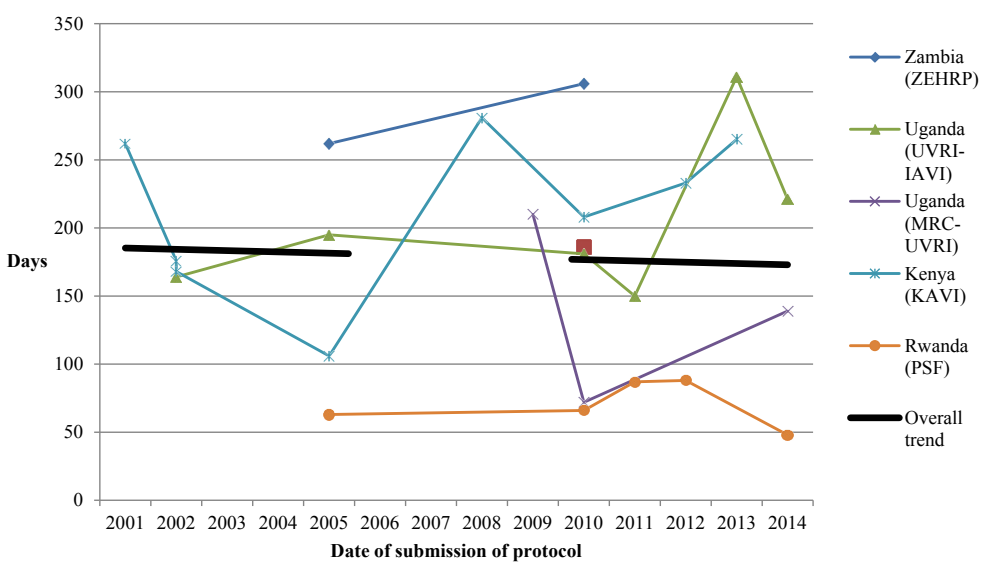

Figure 5: Temporal trends in the approval timelines for regulatory and ethics review for clinical trial protocols at PSF, ZEHRP, Aurum Institute, MRC-UVRI, UVRI-IAVI and KAVI-ICR.

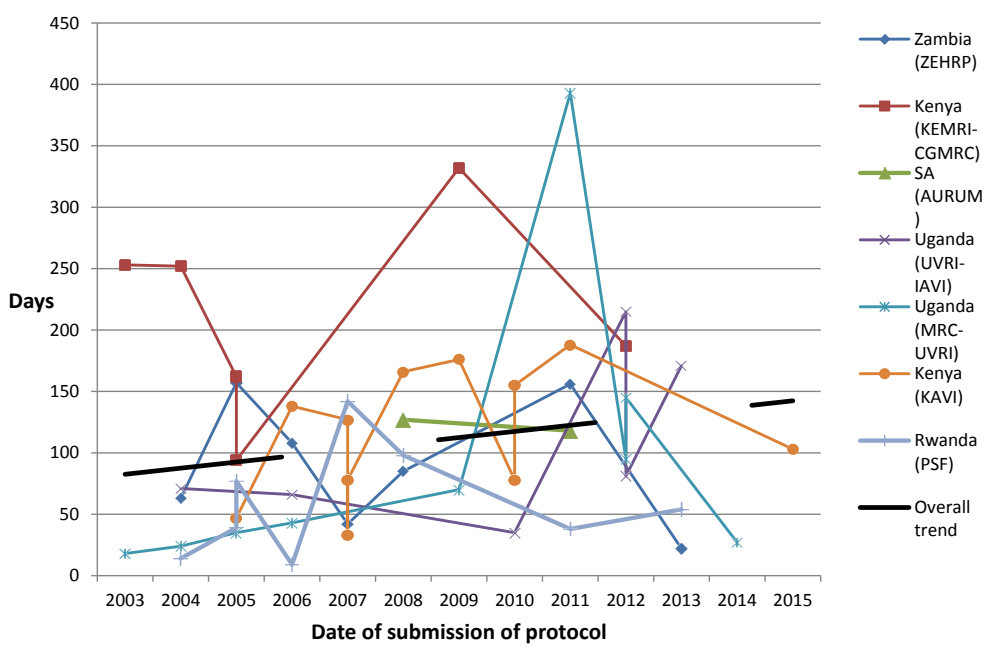

Figure 6: Temporal trends in the approval timelines for regulatory and ethics review for epidemiological study protocols at PSF, ZEHRP, Aurum Institute, MRC-UVRI, UVRI-IAVI, KEMRI-CGMRC and KAVI-ICR.

Overall, factors that contributed to trend in reduction of review timelines (at least according to CRC directors and persons in charge of the IRB docket at these CRCs), included the consolidation of two or more review institutions into one and thus the reduction of the number of review steps that must be fulfilled prior to approval being granted. For instance, this factor was cited by respondents for KEMRI-Welcome Trust Research Programme: 
"The merger of the scientific steering committee (SSC) and the ethics review committee (ERC) to form the scientific and ethics review committee (SERU) in 2015 has reduced the review period from approximately 4-6 months to about 1-2 months" (KEMRI-Wellcome Trust Research Programme respondent).

Another factor that has been associated with reductions in approval timelines is capacity building of ethics review committees (ERCs) in recruitment of additional staff, training of personnel and provision of allowances to ERC staff. This factor was cited by respondents at MRC/ UVRI and UVRI-IAVI:

"The ethics committees are more staffed and meet more regularly. The committee members have also had relevant support from UVRI in terms of allowances. Training of members has also been part of the improvement process" (MRC/UVRI respondent).

"The Institutional Review Committee (IRC) and regulatory are now more experienced and are less burdened because more review boards members have come on board" (UVRI-IAVI respondent).

The provision of standardized protocol submission formats and standard operating manuals (SOPs) that facilitate the submission of complete protocols for review has also been mentioned as a factor that has contributed to a reduction in approval timelines. This factor was mentioned by respondents from ZEHRP and Aurum Institute:

“...There are standard formats for protocol submission and these have been made available and they enable the applicants to fulfill all the requirements and avoid unnecessary delays due to incomplete submissions...' (ZEHRP respondent).

"The ethics committee has provided guidelines and SOPs on submission processes which have helped streamline the application process" (Aurum Institute respondent)"

The prescription of limits to approval timelines in guidelines was also submitted as a factor associated with reduction in approval timelines:

"UNZABREC ensures that response is given from the first review by the second week of the following month, i.e., about one month after submission" (ZEHRP respondent)."

Qualitative feedback on factors (issues) that need to be addressed to reduce approval timelines of ethical and regulatory review of clinical trial and epidemiological study protocols

Responses from the CRCs suggested that there are several factors (issues) that need to be addressed in order to reduce approval timelines of ethical and regulatory review of clinical trial and epidemiological study protocols. One such issue relates to capacity strengthening of review institutions by increasing the number of reviewers especially those with expertise in HIV vaccines:

"Reviewers are part time and usually overwhelmed with the number of studies to review. The Zambian IRB at UNZABREC could benefit from more reviewers. When experts in the research proposed are missing, UNZABREC should feel free to contract reviewers local and international that better understand the field of research proposed" (ZEHRP respondent).

The adoption of parallel rather than sequential approval processes was also submitted as an issue that needs to be addressed in order to reduce approval timelines:
"There are currently no major issues on the structure of the ethical/ regulatory submissions and approvals in Rwanda, except the lack of flexibility to do simultaneous submissions to the existing review bodies. Submissions are required to be performed consecutively as it is outlined in the researchers' guidelines in the health sector" (PSF respondent).

The need for standardized formats for the preparation and submission of protocols was highlighted as an issue that needs to be addressed in order for protocols to be submitted with insufficient information. Further, the need for these standardized formats to be aligned with guidelines provided by ethics committees was mentioned:

"CRCs need to ensure that protocols and relevant participant information sheets and informed consent documents are in alignment with guidelines provided by the ethics committee. Most queries raised are related to insufficient information..." (Aurum Institute respondent).

The need for electronic platforms and databases for the management of the protocol submission and review processes was mentioned by respondents from MRC-UVRI:

"I would like there to be a database managed by regulatory bodies where everyone following up on any submission can check and get to know the status of their submission and how long they should be waiting" (MRC/UVRI respondent).

\section{Discussion}

This study is one in a few systematic evaluations of approval timelines of regulatory and ethical review processes for HIV clinical trials and epidemiological studies in Africa. We show that on average it takes 178 days (approximately 6 months) and 108 days (approximately 4 months) to obtain full authorization to commence HIV clinical trials and epidemiological studies respectively. This study suggests that approval timelines for HIV clinical trials in Africa may be shorter than other AIDS clinical trials as suggested by earlier studies. For instance, a survey of approval timelines at 23 research sites that were part of the AIDS Clinical Trials Group (ACTG) that investigated 21 protocols approved between 2004 and 2012 in 12 countries in Africa, Asia, South America and the Caribbean reported that approval timelines had an average of 17.84 months and ranged from 3 to 37 months [7].

However, comparison of approval timelines for HIV vaccine clinical trials to other vaccines such as TB vaccine trials in Africa suggests that HIV vaccine trials may have longer approval timelines. For instance, Geldenhuys et al. quantified the time taken to achieve ethical and regulatory approval of TB vaccine trials at the South African Tuberculosis Vaccine Initiative (SATVI) and found that the median approval time for protocols submitted to the Medicines Control Council (MCC) and the University of Cape Town (UCT) Faculty of Health Sciences Human Research Ethics Committee (HREC) was 122 and 60 days, respectively [8]. These findings suggest that TB vaccine clinical trials in South Africa experience shorter approval timelines compared to HIV vaccine trials in Eastern Africa and Zambia.

In comparison to more developed economies; we found that approval timelines for HIV clinical trials in Africa are longer. For instance, while it takes 178 days to secure approval for HIV clinical trials in Africa, approval timelines for clinical trials in general (i.e., clinical trials of vaccines as well as other classes of pharmaceutical products) in the USA and the EU are estimated at 15-45 days and 43-75 days, respectively [9-12].

Our study also revealed that approval time-lines vary significantly by country and can range from 70 days in Rwanda to 284 days on 
Zambia. Further, our analysis reveals that there are differences in approval timelines in different research centers even in cases where the research centers are conducting the same HIV vaccine clinical trial. The existence of significant variations in approval timelines across countries and clinical research centers has been observed by earlier studies. In the study of approval timelines for TB vaccine clinical trials, Geldenhuys et al. found that there were variations in approval timelines of ethical and regulatory review processes across research centers and even in the case where protocols were submitted by different research centers to the same review organization. In the study by Geldenhuys et al., there was up to a five-fold variation in approval times across 16 protocols submitted to Medicines Control Council in South Africa for TB vaccine trials. Nonetheless, according to Geldenhuys et al., variations and thus unpredictability of approval process, regardless of the cause, makes planning difficult for researchers and creates anxiety among sponsors/ funders of vaccine trials [8]. Particularly in multi centre studies.

Our analysis of temporal trends in approval timelines for HIV vaccine clinical trials suggests that, in general and taking all CRCs together and without taking into account study differences, there is no evidence of a change in approval timelines between 2001 and 2015. This finding is in line with the study approval timelines of TB vaccine clinical trials conducted by Geldenhuys et al. In that study by Geldenhuys et al. [8] which investigated trials initiated between 2004 and 2012, it was reported that there was no significant difference in approval time by year of submission - an observation that seems to suggest that there may be no evidence of a change in the timelines of ethical and regulatory approval processes related to TB vaccine trials in South Africa over the study period. Interpretation of these findings on temporal trends in approval timelines needs to be done with caution considering that approval timelines may be affected by many factors at play in different countries at different time points that may explain the observation that there was no significant difference in approval time by year of submission. These factors include: financial and human resources available to review boards in different countries at different time points $[13,14]$; capacity building structures of RECs in different countries [7] and complexity of study protocols submitted for ethical and regulatory review. In light of these factors and the fact that the analysis of temporal trends presented by our study and that by Geldenhuys et al. [8] does not account for these potentially confounding factors, the interpretation of these findings from the analysis of temporal trends should be done with caution.

Similarly, detailed comparison of specific HIV vaccine clinical trials across individual CRCs and countries revealed that approval trials for clinical trials conducted at PSF are shorter compared to approval timelines for the same trials conducted in other CRCs. These salient differences strongly suggest that assessment of approval timelines for HIV vaccine trials should be granular and take account of country specific factors. Our analysis of approval timelines in the different CRCs and countries suggest that countries with sequential, rather than parallel, submission procedures had longer approval timelines (with the exception of Rwanda). Further, clinical trial approval timelines that were longer than 200 days were associated with amendment submissions prior to initial approval, investigational products that required institutional biosafety committee review or factors that are peculiar to individual studies such as the loss of protocol amendments during the review process. Lengthier reviews were also associated with epidemiological studies with more invasive procedures.

The respondents in this study submitted several factors that affect ethical and regulatory approval timelines. For instance, the influence of building capacity of ERCs on approval timelines that was highlighted by one of the CRCs investigated here has also been described in African countries [7] as well as in other countries such as Turkey [15] and Pakistan [16]. Several strategies that can contribute to reductions in approval timelines were suggested by the CRCs investigated in this study. These strategies included: the use of electronic platforms and databases for the management of the protocol submission and review processes; adoption of parallel rather than sequential approval processes; capacity building of review institutions in terms of the need for more reviewers especially those with expertise in HIV vaccines; adoption of standardized formats for the preparation and submission of protocols; and the prescription of limits to approval timelines in legislation and/or guidelines used by review organizations.

There is some evidence in literature that these strategies can indeed contribute to reductions in approval timelines. For instance, the use of information technology (IT) to develop web-based platforms for ethical and regulatory process management has been shown to reduce approval timelines. Independent assessment of the Research for Health and Innovation Organizer (Rhinno) Ethics - a web-based platform that automates the entire life cycle of ethical and regulatory review processes [17] revealed that, on the basis of feedback from the early adopters of the platform, the use of the platform has resulted in a $57 \%$ reduction in approval timelines for REC that had been using the platform for more than 2 years. This observation suggests that the use of IT is a plausible strategy to improve approval timelines. The electronic platform has also enabled standardization of formats for the preparation of submission of protocols. This may be particularly important considering that a majority of the queries raised by review institutions were due to either missing or insufficient information in the submitted protocols - a problem that can be addressed by the use of standard templates for protocol submission that make it mandatory for applicants to submit all required information and avoid unnecessary delays due to incomplete submissions.

The adoption of parallel rather than sequential approval processes has also been shown in literature to be associated with reduction in approval timelines [18]. In this light, adoption of parallel approval processes is a promising strategy for the reduction of approval timelines in Africa.

While the prescription of the limits to approval timelines in legislation and/or guidelines used by review organizations was submitted as a strategy that can reduce approval timelines, literature on this suggests otherwise. In Africa, the Pharmacy and Poisons Board of Kenya, for instance, prescribes that decisions on applications to conduct clinical trials should be given within 30 days of application [19]. In Nigeria, institutional health research ethics committees have to communicate decisions on clinical trial applications within 3 months of receipt of applications [20]. We did not find any evidence on the impact of these guidelines on the ethical and regulatory approval timelines in Kenya and Nigeria. Outside Africa, the prescription of limits to approval timelines has been applied in the EU. The EU adopted the Clinical Trials Directive (EUCTD) in 2001 in a bid to improve the clinical research process [21]. The directive aimed to streamline approval process for all clinical trials by defining the role of central and local research ethics committees, allowing parallel and not sequential submission of protocols to several review institutions and outlining that these institutions provide decisions on submitted protocols within 60 days. An analysis of the impact of this directive revealed that there was no significant difference in approval times between EUCTD compliant research centers and centers following local legislation [8]. 
These findings from literature, coupled by the observation that approval timelines are longest in Zambia (284 days on average) yet approval time limits have been prescribed in the guidelines used by Zambia's ethics review committee strongly suggest that the prescription of approval limits in legislation or guidelines is not a panacea and an effective strategy to reduce approval timelines in Africa.

\section{Limitations of the study}

This study focused on HIV Vaccine timelines sponsored by IAVI. While IAVI sponsored studies represent one of the longest and largest HIV vaccine and epidemiologic research in Eastern and Southern Africa, it does not represent the timelines for other HIV clinical trial networks. The qualitative analysis on factors affecting timelines and areas of improvement focused on the researchers' perspectives only and did not include perspectives from regulators and ethics review committees. This study did not also collect comparative country data on other confounding factors such as number or quality of human resources in regulatory bodies or ethics committees that could moderate and explain differences in review timelines.

\section{Conclusion}

Approval timelines for IAVI HIV vaccine clinical trials and epidemiological studies in Eastern and Southern Africa average at 178 and 108 days respectively. Approval timelines for HIV vaccine clinical trials are shorter than the average found for AIDS clinical trials in Africa but seem longer than approval timelines for clinical trials in other regions such as the USA and the European Union. While approval timelines in Africa vary across countries and are multifactorial, countries with sequential, rather than parallel, submission procedures tended to have longer review timelines. Other key factors affecting review timeline included delays associated with amendment submissions prior to initial approval, investigational products that required institutional biosafety committee review or novelty of a product or procedures.

This study recommends that, in order to realize reduced approval timelines in Africa, there is need to adopt and enhance the use of electronic platforms and databases for the management of the protocol submission and review processes; implement parallel rather than sequential ethical approval processes; build capacity of review institutions in terms of the number and HIV vaccine-related expertise of reviewers.

\section{Acknowledgement}

We wish to thank the CRCs described in this paper for in the study; the Pls and persons in-charge of the IRB dockets at the CRCs for their time and insight. Special thanks to all Principal Investigators and CRC staff that provided detailed data and responded to the questionnaires. Special thanks to Frances Priddy, Anatoli Kamali, Pat Fast and Carl Verlinde for their validation of the data, review, edits and guidance on this study.

\section{References}

1. Puppalwar G, Mourya M, Kadhe G, Mane A (2015) Conducting clinical trials in emerging markets of sub-Saharan Africa: Review of guidelines and resources for foreign sponsors 23-34.
2. Macklin R (2004) Double standards in medical research in developing countries. Cambridge University Press.

3. American Health Line (2006) Research notes - Clinical trials: Efforts to increase participation examined.

4. Bollyky TJ, Cockburn IM, Berndt E (2010) Bridging the gap: Improving clinical development and the regulatory pathways for health products for neglected diseases. Clin Trials 7: 719-734.

5. DiMasi JA (2002) The value of improving the productivity of the drug development process: Faster times and better decisions. Pharmacoeconomics 3: 1-10.

6. Miles MB, Huberman AM (1984) Qualitative data analysis: A sourcebook of new methods. SAGE publications Inc., California.

7. Ndebele P, Blanchard-Horan C, Shahkolahi A, Sanne I (2014) Regulatory challenges associated with conducting multicountry clinical trials in resourcelimited settings. J Acquir Immune Defic Syndr 65: 29-31.

8. Geldenhuys H, Veldsman A, Tameris M, Luabeya A, Hanekom W (2012) Analysis of time to regulatory and ethical approval of SATVI TB vaccine trials in South Africa. S Afr Med J. 103: 85-89.

9. Heerspink HJ, Dobre D, Hillege HL, Grobbee DE, De Zeeuw D (2008) Does the European clinical trials directive really improve clinical trial approval time? $\mathrm{Br} J$ Clin Pharmacol 66: 546-550.

10. Mallick AA, O'Callaghan FJ (2009) Research governance delays for a multicentre non-interventional study. J R Soc Med 102: 195-198.

11. Salman RA, Dennis MS, Sandercock PAG,White PM, Warlow C (2007) Research governance impediments to clinical trials: A retrospective survey. J R Soc Med 100: 101-104.

12. Chester P, Alaam S, Cooper P, Risley C, Dornhorst A (2006) Local clinical trial approval times in the UK. International Journal of Pharmaceutical Medicine 20: 317-320.

13. Kass NE, Adnan AH, Ademola A, Appiah-Poku J, Barsdorf N et al. (2007) The structure and function of research ethics committees in Africa: A case study. PLoS Med 4: e3.

14. Blanchard-Horan C, Stocker V, Moran L, Elaine O F, Karin L K et al. (2012) Examining the challenges and solutions to the implementation of trials in resource-limited settings: Limited resource trials. Appl Clin Trials 21: 34-42.

15. Ilgili O, Arda B, Munir K (2014) Analysis of the regulatory bioethical review framework for clinical trials in Turkey. Rev Rom Bioet. 12: 5-14.

16. Jafarey AM, Iqbal SP, Hassan M (2012) Ethical review in Pakistan: The credibility gap. J Pak Med Assoc 62: 1354-1357.

17. http://africa.cohred.org/rhinno-ethics-video

18. Duley L, Antman K, Arena J, Avezum A, Blumenthal M, et al. (2008) Specific barriers to the conduct of randomized trials. Clin Trials 5: 40-48.

19. Pharmacy and Poisons Board, Kenya (2016) Guidelines for applications to conduct clinical trials in Kenya.

20. National Agency for Food and Drug Administration and Control (2016) Regulatory and Registration Directorate: Documentation guidelines for clinical trials in Nigeria. National Agency for Food and Drug Administration and Control, Abuja, Nigeria.

21. Directive 2001/20/EC of the European Parliament and of the Council of 4 April 2001 on the approximation of the laws, regulations and administrative provisions of the Member States relating to the implementation of good clinical practice in the conduct of clinical trials on medicinal products for human use (2001) J Eur Commun 121: 34-44. 